\title{
Coordinated scheduling model of power system with active distribution networks based on multi-agent system
}

\author{
Jingzhe HU $^{1}$, Hao CONG ${ }^{1}$, Chuanwen JIANG ${ }^{1}$
}

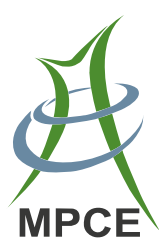

\begin{abstract}
With the large-scale development of distributed generations (DGs) and the connection into the main grid of active distribution networks (ADNs), traditional centralized dispatch of power system has encountered enormous challenge. In a bilateral electricity market, introducing ADN resources in the day-ahead generation schedule will not only enrich the dispatch patterns to the power system, but also reflect the initiative of ADNs. This paper proposes a coordinated scheduling model of power system with a plurality of ADNs based on multi-agent system where ADN agents are brought in the day-ahead market clearing. The process of market clearing and the dispatch of DGs in ADNs are independent with each other but linked together through the market clearing price (MCP) and bid volume. The optimal operating point of the whole system is achieved through multiple information exchange. In comparison with the dispatch without interaction between ADNs and the market operator (MO), the coordinated scheduling model is applied in a system with four ADNs to verify that the proposed method can improve the overall interests of ADNs. Finally, the effects of storage device and tie-line power limit are analyzed.
\end{abstract}

CrossCheck date: 3 August 2017

Received: 17 November 2015/Accepted: 3 August 2017/Published online: 1 November 2017

(c) The Author(s) 2017. This article is an open access publication

$\triangle$ Chuanwen JIANG

jiangcw@sjtu.edu.cn

Jingzhe HU

jingzhehu@yeah.net

Hao CONG

babyconghao@gmail.com

1 School of Electronic Information and Electrical Engineering, Shanghai Jiao Tong University, Shanghai 200240, China
Keywords Multi-agent system, Active distribution network, Market clearing price (MCP), Coordinated scheduling

\section{Introduction}

With the gradual deterioration of global environmental problems and exploration of fossil fuels, large numbers of distributed generations (DGs) are connected to the distribution network. Although this phenomenon will bring environmental and economic benefits to power producers, the operation and scheduling of power system will be faced with significant challenges [1]. As a result, the traditional unidirectional power flow of passive power grid has failed to adapt to the significant changes. Thus the concept of active distribution network (ADN) is put forward [2, 3]. CIGRE C6.11 working group defines ADNs as distribution networks that have systems in place to control a combination of distributed energy resources (generators, loads and storage). Distribution system operators (DSOs) have the possibility of managing the electricity flows using a flexible network topology. Distributed energy resources (DERs) take some degree of responsibility for system support, which will depend on a suitable regulatory environment and connection agreement [4]. From then on, Active distribution network has received extensive attention. Many scholars began to study the generation schedule in ADNs to improve the acceptance level of DG resources, such as optimal power flow [5], reactive power optimization [6], day ahead and real-time scheduling [7], active and reactive power coordinated schedule $[8,9]$. On the other hand, coordinated dispatch between ADN and transmission network (TN) has become a new issue, which is also the topic of this article. 
Centralized and decentralized optimizations are two main methods to solve the multi-area coordination problem. Centralized optimization [10-12] is abandoned gradually because of the large computational quantity and unavailable access to a large amount of generator information. For one thing, it is hard for TN operators to get and handle all the DG operating conditions. For another, suppliers with several DGs are unwilling to provide every DG parameter for information privacy. Decentralized optimization includes Lagrangian relaxation, Benders decomposition and optimality condition decomposition (OCD). The main idea of Lagrangian relaxation is to add shared constraints to each subproblem and update multipliers [13]. Augmented Lagrangian based decomposition [14-18] is the extended method of Lagrangian relaxation and used widely in multi-area optimal power flow (OPF). Benders decomposition is first proposed in [19]. Compared to Lagrange relaxation, Benders decomposition doesn't need to modify the multipliers to get a good convergence rate. Intelligent algorithms such as particle swarm optimization [20], artificial bee colony optimization [21], teaching learning based optimization [22] are also often used for multi-area optimization involving nonlinear conditions such as valve point loading effects. Another widely used method is OCD, which is first proposed in [23] and improved in [24] and [25]. In [26], OCD is implanted on a network of workstations considering physical constraints. References [27] and [28] apply OCD in alternating current (AC) conditions. Tie-line information such as power, price and voltage is exchanged between subsystems, which realizes system decomposition and interaction. OCD does not need to carry out complex formula conversion which is essential in Lagrange relaxation and Benders decomposition.

However, [13-28] are focused on coordinated optimization between TNs and subsystems are parallel to each other. So all sub optimization problems use the variables of same nature, which does not apply to coordinated scheduling problem between ADNs and TN. From the TN side, ADNs is viewed as load and tie-line power can affect TN generation schedule. TN is a virtual source for ADNs and the electricity price influences ADN dispatch. It is necessary to realize the coordinated scheduling between TN and ADNs according to this characteristic.

References [29-31] focus on the coordinated scheduling problem between TN and ADNs. Reference [29] makes the tie-line power difference of TN and ADN optimization as a penalty term and adds it to the objectives of each subproblem, which guarantees the convergence of the algorithm. Price change is not considered in the ADN dispatch. $\mathrm{TN}$ and ADNs are in a parallel relationship which is not suitable. Heterogeneous Decomposition is proposed in $[30,31]$. Power fee calculated by node price is added to the
ADN cost. Nodal price sensitivity matrix is used to improve the convergence speed of the algorithm. Reference [30] verifies the convergence of the algorithm. Reference [31] puts forward two methods for the price sensitivity matrix. This method is based on economic dispatch and node price and not applicable in the electricity market cleared by the uniform market clearing price (MCP).

In the OCD, each subsystem is autonomous and all subsystems interact with each other only through the tieline information, which is the same as the characteristic of agents. Inspired by the concept of OCD, this paper intends to use multi agent system to solve the coordinated scheduling problem between TN and ADNs.

Multi-agent system (MAS) has been applied in the field of electricity market incorporating DGs. Reference [32] researches on EV aggregation scheduling strategy to participate in the power market. The dispatch process is single direction and without taking into account of the impact of EV behavior on the power market. The influence of demand response on the bidding strategies of conventional generators is studied in [33]. In [34], MAS is used to study the bidding strategy of wind power supplier. The impact of other participants is not considered in the model. In [35], a bi-level stochastic model is established to discuss the dispatch strategies in electricity markets. Electric vehicles, active load and wind turbine are collected as different DG suppliers. Therefore, no literatures about MAS study on the topic that ADN acts as an aggregator of various DGs and takes part in the power market.

The contributions of this paper are:

1) Different DGs in the ADN are collected together as a price taker and take part in the power market. ADN can adjust the amount of power bought from the TN to minimize its operating cost through DG generation alterations.

2) A coordinated scheduling model based on MAS for power system with ADNs is proposed. The model of market operator (MO) agent is a day-ahead market clearing problem in the bilateral electricity market while ADN dispatch is a coordinated scheduling model of DGs.

3) Coordination between $\mathrm{ADN}$ and $\mathrm{TN}$ is simulated in the bi-directional mode. MCP is an incentive of the TN to influence the dispatch of DGs in ADNs. ADNs affect the operating of $\mathrm{TN}$ by determining the bid volume. ADNs interact with each other indirectly by affecting the MCP together. Feedbacks of the ADN and TN are achieved by the iteration.

The reminder of this paper is structured as follows. Section 2 introduces the bidding structure based on MAS. Models of every agent and the procedure of coordinated schedule are proposed in Section 3. Section 4 presents 
numerical examples and results. Conclusions are drawn in Section 5.

\section{Bidding structure based on MAS}

\subsection{Interaction mechanism between ADNs and main grid}

In conventional power system, distribution network $(\mathrm{DN})$ is passive and only transmits and distributes power to the end-users. The generation dispatch schedule for the whole power system is the plan just for generators of main grid side. However, as large numbers of DGs connect into DN, DN gradually converts its role from passivity to initiative. Power flow in power system has significant changes and the generation dispatch includes generator scheduling in each autonomous system. However, the main grid and DN together as a whole power system, changes of main grid affect the operation and schedule of $\mathrm{DN}$, and vice versa. Therefore, multiple interactions and generation schedules between the main grid and ADNs are required in order to reach the optimization of the whole system.

Smart grid has the inherent characteristics of informatization, digitization, automation and interactivity, which makes it possible to exchange information frequently between ADNs and the main grid. With technical support of smart grid, a coordinated scheduling model is proposed between the main grid and ADNs in a bilateral electricity market. The market-clearing process of this model is a process of multiple iterations. Interaction between ADNs and the main network is achieved mainly through information exchange among MO agent and ADN agents. Brokered deal and network security check are carried out in the MO agent based on bid volumes offered by ADN agents, through which MCP is determined. According to $\mathrm{MCP}$ provided by $\mathrm{MO}$ agent, each $\mathrm{ADN}$ agent optimizes the dispatch of DGs and re-declares the bid volume of electricity to the MO agent. By repeated exchanges of MCP and bid volumes between the two sides, a market equilibrium is achieved ultimately.

\subsection{Market framework}

According to the characteristics of MAS, each agent represents a separate physical entity and can coordinate with other agents. The market framework based on MAS consists of MO agent, ADN agent, DG agent, buyer agent and supplier agent. The market framework is shown in Fig. 1.

MO agent: market clearing, determining the MCP and power volume of both buyer agents and supplier agents,

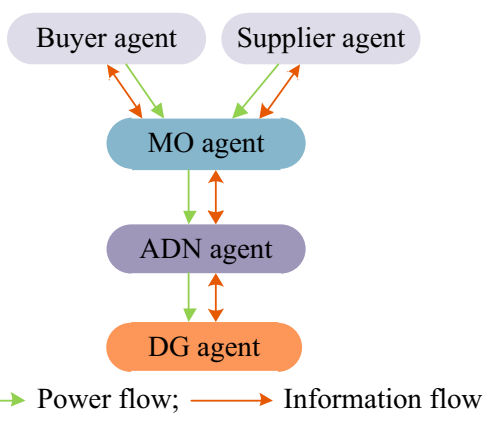

Fig. 1 Market framework

sending these messages to other agents, network security check in the main grid.

Buyer agent: making bidding strategy, providing bidding curve to MO agent, bidding strategy adjustments.

Supplier agent: making bidding strategy, offering biding curve to MO agent, bidding strategy adjustments.

ADN agent: providing bid volume to $\mathrm{MO}$ agent, generation dispatch of DGs based on MCP sent by MO agent and parameters provided by DG agents, network security check in the ADN.

DG agent: generation in accordance with the command of ADN agent, sending cost and operating data to ADN agent, operating security check of the DG.

JADE is employed as a multi-agent framework in this paper. The communication among agents is carried out according to FIPA-specified agent communication language (ACL). The structure of ADN agent is shown in Fig. 2. The dispatch model proposed in this paper is set in the information processor. Environment affects the ADN agent through perceptron. ADN agent interacts with other agents through communication mechanism.

After registering a unique ID and defining the behavior for each agent, MAS can handle the coordinating problem between TN and ADNs through information exchange and control strategies. The coordination control of MAS

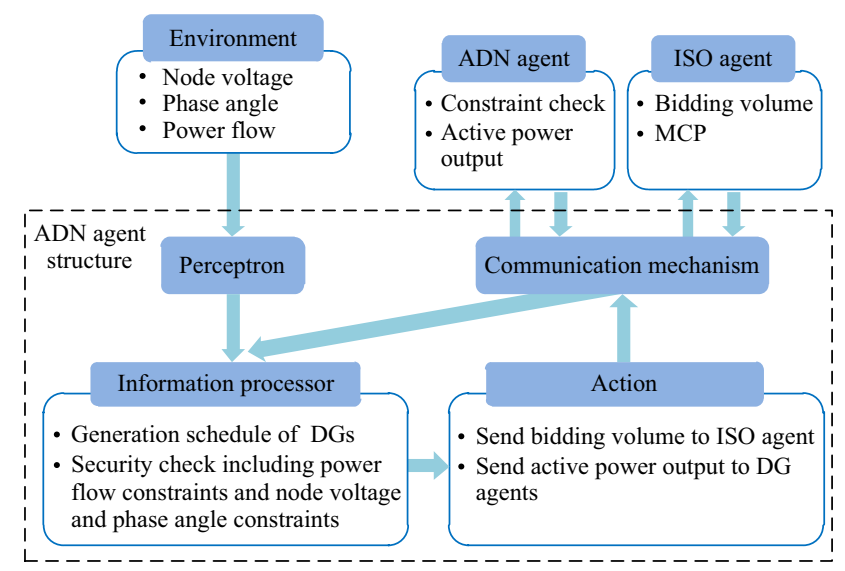

Fig. 2 Structure of ADN agent 
includes the definition of ADN bid volume and the market clearing progress.

The definition of ADN bid volume is as follows.

1) $\mathrm{ADN}$ agent drafts $\mathrm{DG}$ generation strategies, define the power output of each DG, and send 'request' message to DG agents.

2) DG agents receive messages from the ADN agent and assess the security conditions.

3) If the output proposal from ADN agent is judged available by the power and state of charge(SOC) index, send 'agree' message to the ADN agent.

4) $\mathrm{ADN}$ agent sends 'propose' message to all DGs to implement the generation schedule.

5) If the security constraints in DG agents are violated, return 'refuse' message to the ADN agent.

6) $\mathrm{ADN}$ agent makes adjusting strategies and re-sends 'propose' message to all DGs to implement the generation schedule.

7) After every DG agent returns message, the DG generation schedule is determined.

The market clearing progress is as follows:

1) Market clearing is made in the MO agent and define the MCP. MO agent sends 'inform' message to ADN agents.

2) ADN agents determine DG coordinated schedule and send bid volume 'request' message to the MO agent.

3) MO agent assess the power difference index and send 'agree' or 'refuse' message to ADN agents.

4) If $\mathrm{ADN}$ agents receive 'refuse' message, coordinated schedule is adjusted and 'request' messages are re-sent to the MO agent.

5) If $\mathrm{ADN}$ agents receive 'agree' message, the market clearing results are implemented.

As is can be seen, the core of the coordinated scheduling is the adjusting dispatch strategies of the agents, which is discussed in Section 3.

\section{Coordinated scheduling model of power system with ADNs}

\subsection{DG agent}

DG agent is the agent of DERs in ADNs such as wind turbine (WT) agent, hydropower agent, combined heat and power (CHP) agent and energy storage (ES) agent.

1) WT agent

WT agent is the agent of a wind turbine. Based on predicted wind speed, the equation of active power output is shown as follows [36].

$$
\begin{cases}P_{w}=0 & v_{c o}<v<v_{c i} \\ P_{w}=P_{r}\left(A v^{2}+B v+C\right) & v_{c i}<v<v_{r} \\ P_{w}=P_{r} & v_{r}<v<v_{c o}\end{cases}
$$

where $P_{w}$ is the power output of WT; $v$ is the real wind speed of WT; $v_{r}, v_{c i}, v_{c o}$ is the rated/cut-in/cut-out wind speed of WT; $P_{r}$ is the rated power of WT. The coefficients $A, B$ and $C$ can be formulated as follows.

$$
\begin{aligned}
& A=\frac{1}{\left(v_{c i}-v_{r}\right)^{2}}\left[2-4\left(\frac{v_{c i}+v_{r}}{2 v_{r}}\right)^{3}\right] \\
& B=\frac{1}{\left(v_{c i}-v_{r}\right)^{2}}\left[\frac{\left(v_{c i}+v_{r}\right)^{4}}{2 v_{r}^{3}}-\left(3 v_{c i}+v_{r}\right)\right] \\
& C=\frac{1}{\left(v_{c i}-v_{r}\right)^{2}}\left[v_{c i}\left(v_{c i}+v_{r}\right)-4 v_{c i} v_{r}\left(\frac{v_{c i}+v_{r}}{2 v_{r}}\right)^{3}\right]
\end{aligned}
$$

\section{2) Hydropower agent}

In this paper, we only discuss runoff hydropower plant. Hydropower agent is the agent of hydropower units. The active power output constraint is described in (5).

$0 \leq P_{\text {water }}^{t} \leq P_{\text {water, } \text { max }}^{t}$

where $P_{\text {water }}^{t}$ is the power output of hydropower turbines (HT) at period $t$. Generally, $P_{\text {water, } \max }^{t}$ is constant during a scheduling period (24 hours).

\section{3) CHP agent}

CHP turbine has a rated technical parameter: heat-power ratio $\theta$, which is formulated as follows [37].

$\theta=\frac{Q^{t}}{P_{C H P}^{t}} \times 100 \%$

where $Q^{t}$ is the thermal production of CHP at period $t$; $P_{C H P}^{t}$ is the power output of CHP at period $t$.

The thermal supply constraint of CHP system is shown in $(7)$.

$Q^{t}=Q_{\text {load }}^{t}$

where $Q_{\text {load }}^{t}$ is CHP cooling or heating demand at period $t$.

According to (6) and (7), the active power output can be calculated. Moreover, active power output limit constraint in (8) should be satisfied as well.

$P_{C H P, \min } \leq P_{C H P}^{t} \leq P_{C H P, \max }$

where $P_{C H P, \max }, P_{C H P, \text { min }}$ is the upper/lower limit of active power output of CHP. 


\section{4) ES agent}

The most common ES device is storage battery. The charging and discharging process of a battery is denoted in the following two equations [35].

The charging process:

$S o C^{t}=S o C^{t-1}+P_{c}^{t} \eta_{c}$

The discharging process:

$S o C^{t}=S o C^{t-1}-\frac{P_{d}^{t}}{\eta_{d}}$

where $S o C^{t}$ is the residual capacity of ES at period $t ; P_{c}^{t}$ and $P_{d}^{t}$ is the charging/discharging power of ES at period $t ; \eta_{c}$ and $\eta_{d}$ are the charging/discharging efficiency of ES.

The operating cost can be calculated by (11).

$F_{e s}(t)=e\left(\left|P_{c}^{t}\right|+\left|P_{d}^{t}\right|\right)$

where $F_{e s}(t)$ is the total operating cost of ES at period $t ; e$ is the ES operating cost of per MW.

The operating constrains of ES system is given in (11-7).

$S o C_{\min } \leq S o C^{t} \leq S o C_{\max }$

$-P_{c, \text { max }} \leq P_{\text {storage }}^{t} \leq P_{d, \text { max }}$

$P_{\text {storage }}^{t}=P_{d}^{t}-P_{c}^{t}$

where $P_{\text {storage }}^{t}$ is the power output of ES at period $t ; P_{c, \text { max }}$ and $P_{d, \text { max }}$ are maximum permissible values of ES charging/discharging power.

Constraint (12) and (13) mean the residual capacity and power output of ES limits, respectively. Constraint (14) is the power output formulation.

\subsection{ADN agent}

The generation schedule of DGs is made in the ADN agent, including not only dispatchable units such as hydropower, ES and CHP, but also undispatchable units such as photovoltaic (PV) power generation and WT. The objective function is to minimize the total cost of the ADN:

$\min \sum_{t=1}^{T}\left(\lambda_{A D N}^{t} P_{A D N}^{t}+\sum_{k=1}^{n_{D G}} F_{D G}^{k, t}\left(P_{D G}^{k, t}\right)\right)$

where $\lambda_{A D N}^{t}$ is the MCP at period $t ; P_{A D N}^{t}$ is the power bought from the $\mathrm{MO}$ at period $t ; F_{D G}^{k, t}$ is the cost function of DG $k$ in the ADN at period $t ; P_{D G}^{k, t}$ is the active power of DG $k$ in the ADN at period $t ; n_{D G}$ is the number of DGs in the AND; $T$ is the scheduling period.

Subject to scheduling constraints:

$P_{i n}^{m}-V_{m} \sum_{n=1}^{n_{l}} V_{n}\left(G_{m n} \cos \theta_{m n}+B_{m n} \sin \theta_{m n}\right)=0$
$Q_{i n}^{m}-V_{m} \sum_{j=1}^{n} V_{n}\left(G_{m n} \sin \theta_{m n}-B_{m n} \cos \theta_{m n}\right)=0$

$P_{i n}^{m}=P_{G}^{m}-P_{D}^{m}$

$Q_{i n}^{m}=Q_{G}^{m}-Q_{D}^{m}$

$-P_{m n, \max } \leq P_{m n} \leq P_{m n, \max }$

$V_{m, \text { min }} \leq V_{m} \leq V_{m, \text { max }}$

$\theta_{m, \min } \leq \theta_{m} \leq \theta_{m, \max }$

where $P_{i n}^{m}$ and $Q_{i n}^{m}$ are the active/reactive power injection of node $m ; V_{m}$ is the voltage of node $m ; G_{m n}$ is the conductance of the branch from node $m$ to node $n ; \theta_{m n}$ is the phase angle deviation between node $m$ and node $n ; B_{m n}$ is the susceptance of the branch from node $m$ to node $n ; P_{G}^{m}$ and $Q_{G}^{m}$ are the active/reactive power generation of node $m ; P_{D}^{m}$ and $Q_{D}^{m}$ are the active/reactive power demand of node $m$; $P_{m n}$ is the transmission power linking node $m$ and node $n$; $\theta_{m}$ is the phase angle at node $m$.

The first item in (15) is the fees for purchasing electricity from the $\mathrm{MO}$ and the second is the sum of generation cost. Equation (16) and (17) are the node active and reactive power injection balance. Equation (18) and (19) are the node power injection formulation. Constraint (20) means the power flow limit on transmission lines. Constraint (21) and (22) is the node voltage and phase angle limits.

\subsection{MO agent}

Market clearing is made in the MO agent, which is a unit commitment (UC) problem. MCP is used for settling accounts in a pool based bilateral electricity market, which is calculated by the highest bid price of power suppliers succeeding in generation bidding. The objective function is the maximization of social welfare:

$\max \sum_{t=1}^{T}\left(\sum_{i=1}^{n_{b}} C_{b}^{i, t}\left(P_{b}^{i, t}\right)-\sum_{j=1}^{n_{g}} C_{G}^{j, t}\left(P_{G}^{j, t}\right)\right)$

where $n_{b}$ is the number of buyers; $n_{g}$ is the number of supplies; $C_{b}^{i, t}$ is the bid strategy of buyer $i$ at period $t ; C_{G}^{j, t}$ is the bid strategy of supplier $j$ at period $t ; P_{b}^{i, t}$ is the active power of buyers at period $t ; P_{G}^{j, t}$ is the active power of supplies at period $t$.

The constraints are:

$\sum_{j}^{n_{g}} P_{G}^{j, t}=\sum_{i}^{n_{b}} P_{b}^{i, t}+\sum_{l}^{n_{D G}} P_{A D N}^{l, t}$

$I_{G}^{j, t} P_{G}^{j, \min } \leq P_{G}^{j, t} \leq I_{G}^{j, t} P_{G}^{j, \max }$

$P_{b}^{i, \min } \leq P_{b}^{i, t} \leq P_{b}^{i, \text { max }}$ 


$$
\begin{aligned}
& y^{j, t}-z^{j, t}=I_{G}^{j, t}-I_{G}^{j, t-1} \\
& y^{j, t}+z^{j, t} \leq 1 \\
& P_{G}^{j, t}-P_{G}^{j, t-1} \leq R_{u}^{j}\left(1-y^{j, t}\right)+y^{j, t} P_{G}^{j, \min } \\
& -R_{d}^{j}\left(1-z^{j, t}\right)-z^{j, t} P_{G}^{j, \min } \leq P_{G}^{j, t}-P_{G}^{j, t-1} \\
& \left(X_{\text {on }}^{j, t-1}-T_{o n}^{j}\right)\left(I_{G}^{j, t-1}-I_{G}^{j, t}\right) \geq 0 \\
& \left(X_{\text {off }}^{j, t-1}-T_{\text {off }}^{j}\right)\left(I_{G}^{j, t}-I_{G}^{j, t-1}\right) \geq 0
\end{aligned}
$$

where $P_{A D N}^{l, t}$ is the bid volume provided by the $l$ th ADN at period $t ; I_{G}^{j, t}$ is the binary variable to indicate if supplier $j$ is on at period $t ; y^{j, t}$ and $z^{j, t}$ are the start-up/shut-down binary variable to indicate whether supplier $j$ starts up/shuts down at period $t ; P_{G}^{j, \max }$ and $P_{G}^{j, \min }$ are the maximum/minimum power output of supplier $j ; P_{b}^{i, \text { max }}$ and $P_{b}^{i, \text { min }}$ are the maximum/minimum bid volume of buyer $i ; R_{u}^{j}$ and $R_{d}^{j}$ are the ramp-up/ramp-down rate limit of supplier $j ; T_{o n}^{j}$ and $T_{o f f}^{j}$ are the minimum continues up/down time of supplier $j ; X_{o n}^{j, t}$ and $X_{o f f}^{j, t}$ are the continuous up/down time of supplier $j$.

Objective (23) includes the bidding strategies of buyers and suppliers. The bidding price functions are quadratic functions in (33) and (34), which can be transformed into piecewise-linear segments:

$C_{b}^{i, t}\left(P_{b}^{i, t}\right)=\alpha_{b}^{i}\left(P_{b}^{i, t}\right)^{2}+\beta_{b}^{i} P_{b}^{i, t}+\gamma_{b}^{i}$

$C_{G}^{j, t}\left(P_{G}^{j, t}\right)=\alpha_{G}^{j}\left(P_{G}^{j, t}\right)^{2}+\beta_{G}^{j} P_{G}^{j, t}+\gamma_{G}^{j}$

where $\alpha_{b}^{i}, \beta_{b}^{i}, \gamma_{b}^{i}$ are the coefficients of bidding price functions of buyer $i ; \alpha_{G}^{j}, \beta_{G}^{j}, \gamma_{G}^{j}$ are the coefficients of bidding price functions of supplier $j$.

Equation (24) means power balance between suppliers, buyers and ADNs. Equations (25) and (26) denote power output limits of the suppliers and buyers. Equations (27) and (28) are auxiliary functions. Equations (29) and (30) are the ramp-up and ramp-down constraints. Equations (31) and (32) are the continuous up and down time of supplier constraints, respectively.

\subsection{Procedure of coordinated scheduling model based on MAS}

The algorithm flow to solve the coordinated scheduling model of power system with ADNs is illustrated in Fig. 3. The steps are as follows.

Step 1: Set initial values.

Step 2: MO agent makes market clearing, generation schedule.

Step 3: MO agent determines MCP.

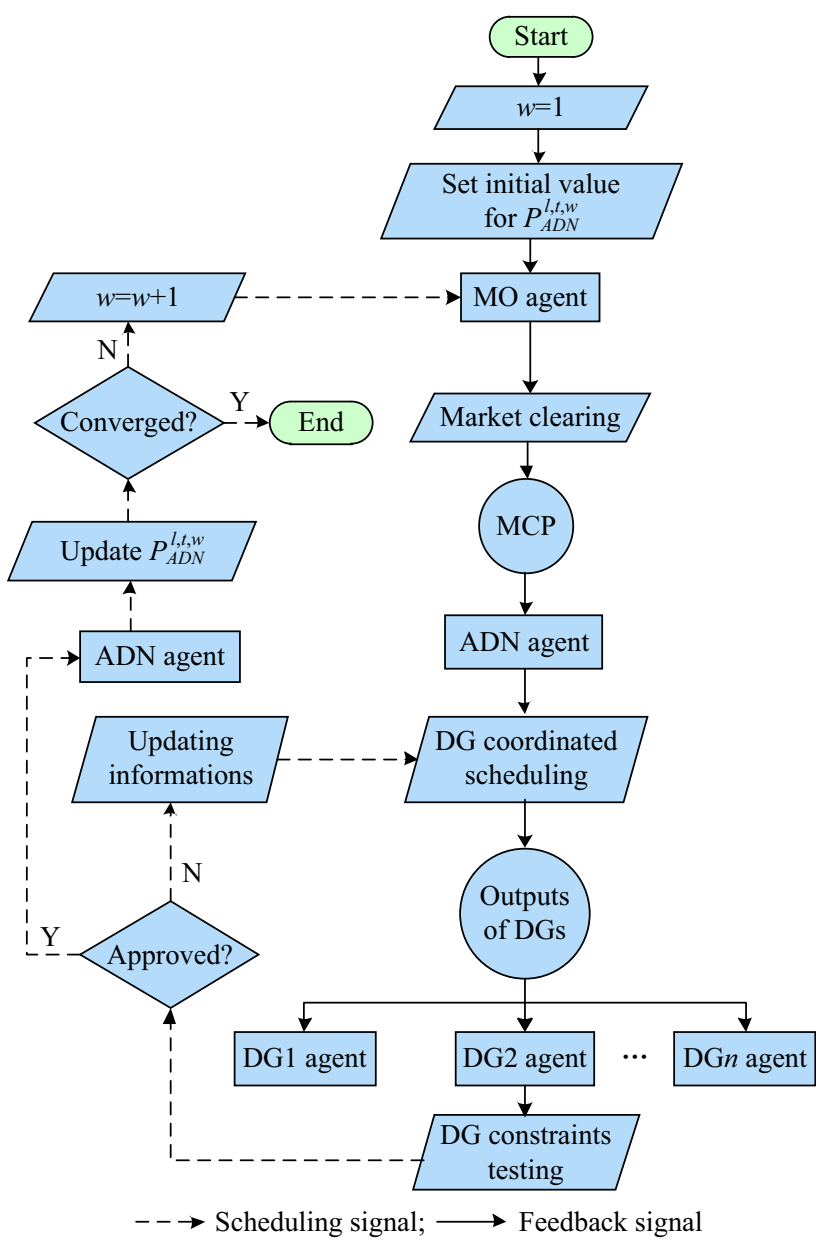

Fig. 3 Algorithm flow chart

Step 4: ADN agent gets MCP, makes the optimal dispatch of DGs and calculates bid volume and power of each DG.

Step 5: DG agent checks the operating security constraints. If constraints are satisfied, turn to Step 6. If not, update DG operating conditions according to (35)-(36) and go back to Step 4.

If the power output constraints of DGs, namely power index $P_{D G}^{k, t}$, are violated, update $P_{D G}^{k, t}$ in the ADN agent as follows.

$P_{D G}^{k, t}= \begin{cases}P_{D G}^{k, \max } & P_{D G}^{k, t}>P_{D G}^{k, \max } \\ P_{D G}^{k, \text { min }} & P_{D G}^{k, t}<P_{D G}^{k, \text { min }}\end{cases}$

If SOC constraints of ES agents, namely SOC index So $C^{t}$, are violated, update $P_{c}^{t}$ and $P_{d}^{t}$ as follows.

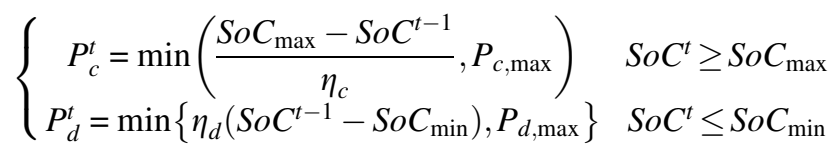


Step 6: ADN agent determines the bid volume $P_{A D N}^{l, t, w}$.

Step 7: ADN agent checks whether the iteration constraint in (37) is satisfied, that is, power difference index $\left|P_{A D N}^{l, t, w}-P_{A D N}^{l, t, w-1}\right|$. Stop if the iteration is converged. Otherwise update the bid volume of ADNs in (24) and go back to Step 2.

$\left|P_{A D N}^{l, t, w}-P_{A D N}^{l, t, w-1}\right| \leq \varepsilon$

where $\varepsilon$ is the iteration error.

\section{Case study}

In this paper, a system with four suppliers, three buyers and four ADNs is applied to test the performance of proposed model. 24-hour reference load, wind speeds, solar outputs and heat demand are given in Table 1 . The bidding information of suppliers and buyers is given in Table 2 . ADN1 has two DGs which are CHP and Gas turbine generator (GT). ADN2 includes 3 DGs which are WT, ES and GT. ADN3 consists of 3 DGs which are PV, ES and GT. ADN4 has two DGs which are HT and GT. ADN1 and
ADN4 have the same topologies and parameters of networks while ADN1 and ADN4 are the same. The topologies of four ADNs are shown in Fig. 4 and Fig. 5. Parameters of networks and DGs for 4 ADNs are given in Tables 3, 4, and 5. The parameters of WT are set as follows: $v_{r}=10.5, v_{c i}=3, v_{c o}=25$. In addition, the generation costs of WT and PV are not included in total costs. Heatpower ratio is 0.9. The load ratio of four ADNs is $\frac{1}{2}: \frac{1}{6}: \frac{1}{6}: \frac{1}{6}$. Rated capacity of ES is $20 \mathrm{MWh}$. The maximum permissible values of charging and discharging power are $2 \mathrm{MW}$. The operating cost of ES is 0.08 .

The convergence error is set to 0.001 . MCP and the tieline power of ADN1 at 17:00 in each iteration is shown in Fig. 6. Figure 7 reveals the convergence performances of social welfare and cost of ADNs. In the first iteration, MO predicts the load amount of ADNs based on historical data and determines a MCP that is higher than the DG operating cost in ADNs. So the output of DGs in ADNs increases, resulting in the decrease of ADN bid volume. Meanwhile, high MCP leads to high ADN cost. The low ADN bid volume lead to a smaller MCP in the second iteration. So ADN bid volume and MCP can affect each other. With

Table 1 24-hour base load, wind speed, solar output and heat demand

\begin{tabular}{|c|c|c|c|c|}
\hline Hour & Load (MW) & Wind speed $(\mathrm{m} / \mathrm{s})$ & Solar output (MW) & Heat demand (MW) \\
\hline 1 & 85 & 9.5 & 0 & 0.16 \\
\hline 2 & 73 & 9.3 & 0 & 0.14 \\
\hline 3 & 65 & 9.1 & 0 & 0.16 \\
\hline 4 & 70 & 8.8 & 0 & 0.18 \\
\hline 5 & 70 & 8.6 & 0 & 0.28 \\
\hline 6 & 88 & 8.3 & 0 & 0.32 \\
\hline 7 & 90 & 8.1 & 0 & 0.40 \\
\hline 8 & 92 & 7.8 & 10 & 0.80 \\
\hline 9 & 99 & 7.3 & 20 & 1.00 \\
\hline 10 & 104 & 6.8 & 40 & 1.08 \\
\hline 11 & 118 & 6.5 & 80 & 0.90 \\
\hline 12 & 122 & 6.6 & 110 & 1.00 \\
\hline 13 & 130 & 7.0 & 180 & 1.08 \\
\hline 14 & 146 & 7.5 & 120 & 1.00 \\
\hline 15 & 149 & 8.2 & 100 & 0.84 \\
\hline 16 & 154 & 8.7 & 110 & 0.72 \\
\hline 17 & 154 & 9.0 & 70 & 0.76 \\
\hline 18 & 148 & 9.2 & 45 & 0.64 \\
\hline 19 & 127 & 9.2 & 10 & 0.50 \\
\hline 20 & 115 & 9.6 & 0 & 0.36 \\
\hline 21 & 109 & 9.8 & 0 & 0.24 \\
\hline 22 & 105 & 9.8 & 0 & 0.20 \\
\hline 23 & 99 & 9.6 & 0 & 0.20 \\
\hline 24 & 96 & 9.5 & 0 & 0.16 \\
\hline
\end{tabular}


Table 2 Bidding information of suppliers and buyers

\begin{tabular}{|c|c|c|c|c|c|c|}
\hline Supplier and buyer & $\alpha\left(\$ / M^{2}\right)$ & $\beta(\$ / M W)$ & $\gamma(\$)$ & $P_{\max }$ & $P_{\min }$ & $T_{o n} / T_{o f f}$ \\
\hline Supplier1 & 0.01532 & 22.5 & 0 & 84 & 25 & $6 / 6$ \\
\hline Supplier2 & 0.00889 & 22.4 & 0 & 95 & 34 & $4 / 1$ \\
\hline Supplier3 & 0.01508 & 26.2 & 0 & 85 & 15 & $1 / 1$ \\
\hline Supplier4 & 0.00208 & 23.9 & 0 & 80 & 39 & $1 / 2$ \\
\hline Buyer1 & - & -0.2 & 56 & 50 & 10 & - \\
\hline Buyer2 & - & -0.25 & 58 & 50 & 5 & - \\
\hline Buyer3 & - & -0.22 & 54 & 50 & 3 & - \\
\hline
\end{tabular}

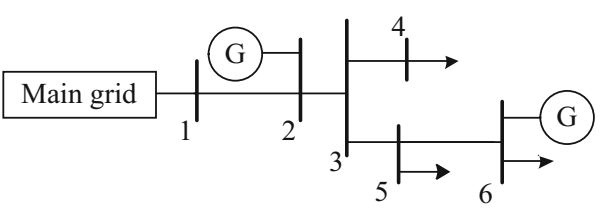

Fig. 4 System topology for ADN1 (ADN4)

Table 3 Network data for ADN1 (ADN4)

\begin{tabular}{llllll}
\hline From bus & To bus & $R$ (p.u.) & $X$ (p.u.) & $B$ (p.u.) & Flow limit (MW) \\
\hline 1 & 2 & 0.1 & 0.2 & 0.04 & 20 \\
2 & 3 & 0.05 & 0.25 & 0.06 & 30 \\
3 & 4 & 0.02 & 0.1 & 0.02 & 30 \\
3 & 5 & 0.12 & 0.26 & 0.05 & 20 \\
5 & 6 & 0.1 & 0.3 & 0.06 & 20 \\
\hline
\end{tabular}

Table 4 Network data for ADN2 (ADN3)

\begin{tabular}{llllll}
\hline From bus & To bus & $R$ (p.u.) & $X$ (p.u.) & $B$ (p.u.) & Flow limit (MW) \\
\hline 1 & 4 & 0 & 0.06 & 0 & 30 \\
4 & 5 & 0.02 & 0.09 & 0.16 & 20 \\
5 & 6 & 0.04 & 0.17 & 0.36 & 20 \\
3 & 6 & 0 & 0.06 & 0 & 30 \\
6 & 7 & 0.01 & 0.1 & 0.21 & 20 \\
7 & 8 & 0.01 & 0.07 & 0.15 & 30 \\
8 & 2 & 0 & 0.06 & 0 & 30 \\
8 & 9 & 0.03 & 0.16 & 0.31 & 30 \\
\hline
\end{tabular}

repeated iteration, ADN bid volume changes smaller and smaller. After 13 iterations, the convergence is reached.

Figure 8 depicts the power output of DGs in ADN1 in a day. CHP always operates with full capacity with low cost. Even though the MCP is high in peak load hours between 15:00 and 18:00, the tie-line power exchange increases because of the high cost of DGs in ADN1.

We consider 4 cases to verify the feasibility of the model. Case 1 is the coordinated schedule in this paper.
Case 2 and Case 3 are cases without interaction between ADNs and MO. The predicted MCP in Case 2 and Case 3 is 0.5 times and 1.5 times of case 1 , respectively. Case 4 is the centralized schedule without ADNs. Table 6 shows the cost and revenue under different cases. From this table, we can see that total cost of ADNs in the three cases without interaction between ADNs and MO is higher than that in Case 1. The volume of power purchased from main grid is the best in the coordinated schedule. No matter more or less the volume is, the cost of ADNs will increase. The cost of per MW in Case 1 is higher than that in Case 4. For the social welfare, it appears on the opposite situation. This means that the centralized schedule can is more economic than the decentralized MAS model and the cost of ADNs is higher. As is mentioned before, the interest of the ADN operators decreases in Case 4 and MO operators can't dispatch DGs in the ADNs in practice.

As a kind of important DERs, energy storage equipment will have a certain impact on the grid scheduling. In order to discuss the influence of energy storage equipment on the dispatch of ADNs, examples with and without ESs are given.

Table 7 gives the cost of ADNs with and without energy storage. The costs of both ADN2 and ADN3 with ES are reduced respectively compared with those without ESs. This is because the energy storage device charges in off-peak hours when the MCP is low and discharges in peak hours when the MCP is high, which reduces the cost of the distribution network. The costs of ADN1 and ADN4 are also changed in the two cases. The usage of ESs affects the bid volumes of ADN2 and $\mathrm{ADN} 3$, which makes the MCP different as well as the fees of purchasing power from the main grid in ADN1 and ADN4. In general, the total cost of the distribution network is reduced due to the influence of load shift of energy storage equipment, which reduces the MCP in peak hours and avoids the start and stop of several units in off-peak hours.

24-hour charging and discharging state of ES in ADN2 is given in Fig. 9. Negative value denotes charging state and positive value denotes discharging state. For ADN2, it 
Table 5 DG data in ADN

\begin{tabular}{|c|c|c|c|c|c|c|}
\hline $\mathrm{ADN}$ & DG & $P_{\min }(\mathrm{MW})$ & $P_{\max }(\mathrm{MW})$ & $a(\$)$ & $b(\$ / \mathrm{MWh})$ & $c\left(\$ / M^{2} h\right)$ \\
\hline \multirow[t]{2}{*}{ ADN1 } & CHP & - & - & 0 & 30 & 0.18 \\
\hline & GT & 0 & 30 & 0 & 25 & 0.15 \\
\hline \multirow[t]{2}{*}{ ADN2 } & WT & 0 & 4 & - & - & - \\
\hline & GT & 0 & 5 & 0 & 36 & 0.11 \\
\hline \multirow[t]{2}{*}{ ADN3 } & PT & 0 & 0.4 & - & - & - \\
\hline & GT & 0 & 30 & 0 & 39 & 0.11 \\
\hline \multirow[t]{2}{*}{ ADN4 } & HT & 0 & 2 & 0 & 40 & 0 \\
\hline & GT & 0 & 5 & 0 & 37 & 0.14 \\
\hline
\end{tabular}

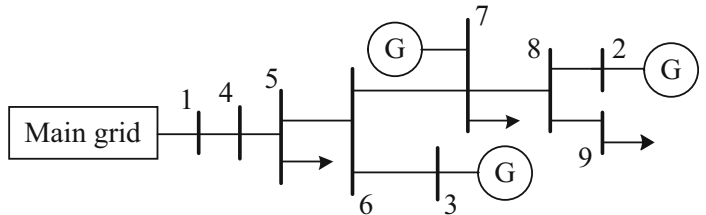

Fig. 5 System topology for ADN2 (ADN3)

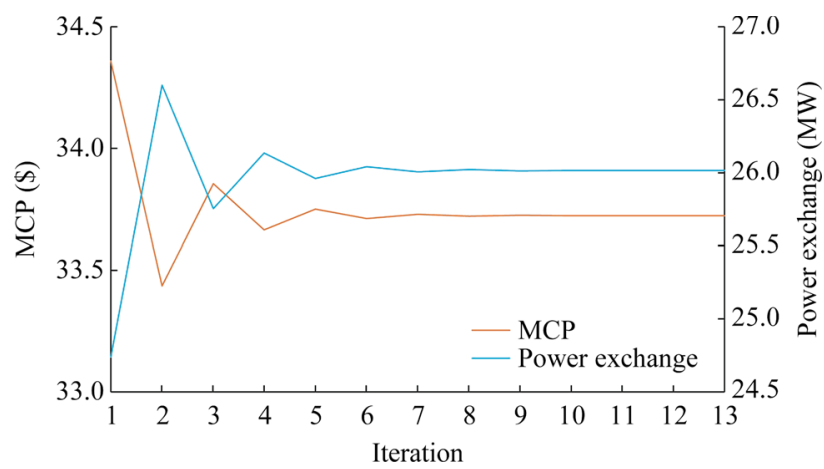

Fig. $6 \mathrm{MCP}$ and power exchange between MO and ADG1 at 17:00

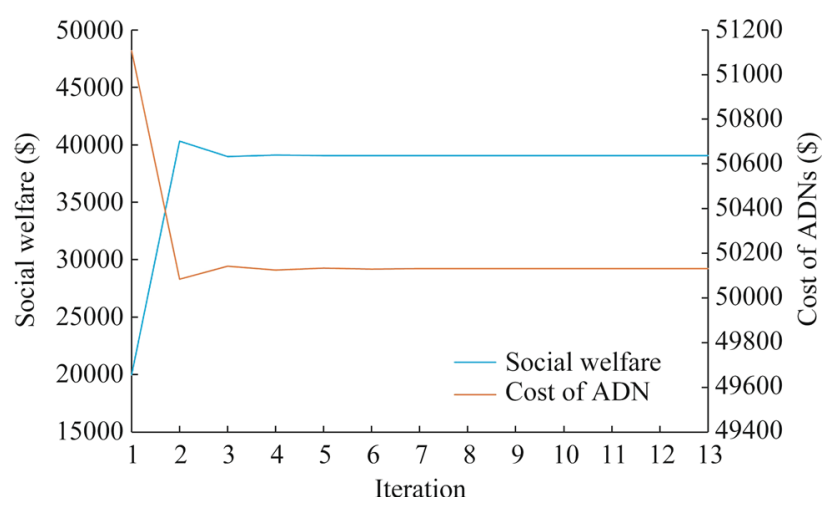

Fig. 7 Convergence performances of social welfare and cost of ADNs

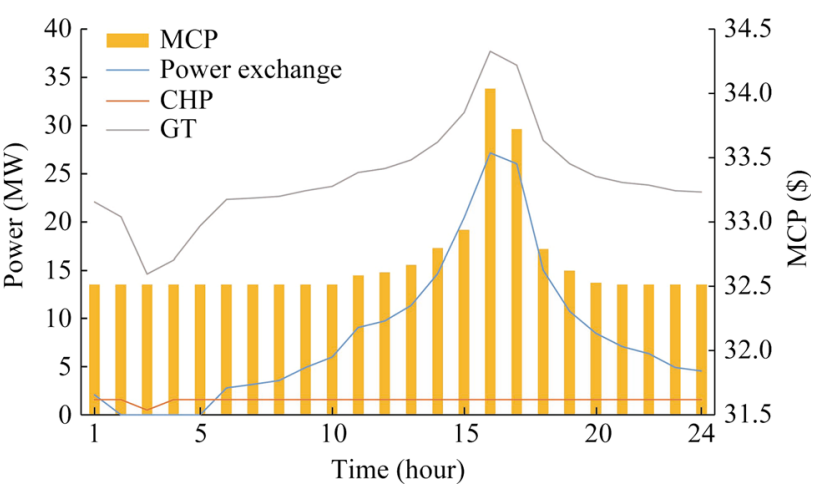

Fig. 8 Power output of DGs in ADN1

can be seen from the Figure 6 that the battery is charged between 1:00 and 7:00 in off-peak hours and discharged between 14:00 and 18:00 in peak hours, which can makes load curve become smoother.

As an important factor to limit the energy interaction between distribution and transmission network, tie-line power transmission limit will affect the dispatch of generation scheduling. Relationship between cost, social welfare and transmission power limit in ADN3 is shown in Fig. 10. From the figure, it can be seen that with the tie-line power cap decreasing, the cost of ADN3 increase and social welfare decreases. This is due to that distribution network cannot use power from the main grid with low cost and can only dispatch units of high cost in ADN3 to meet the load demand.

\section{Conclusion}

Due to the physical connection between ADNs and the main grid, the dispatch of them is a process of mutual influence. Under the smart grid environment, we propose a coordinated scheduling model of power system with ADNs 
Table 6 Cost and revenue under different cases

\begin{tabular}{|c|c|c|c|c|}
\hline Cost and revenue & Case $1(\$)$ & Case $2(\$)$ & Case $3(\$)$ & Case $4(\$)$ \\
\hline Total revenue of suppliers & 180906.20 & 75085.99 & 226492.88 & 212343.20 \\
\hline Total cost of buyers & 149073.00 & 102307.17 & 237144.66 & 165151.90 \\
\hline Cost of per MW & 24.00 & 23.97 & 25.70 & 23.70 \\
\hline Social welfare & 39074.60 & 38767.00 & 29820.00 & 48743.00 \\
\hline Cost of ADN1 & 25302.95 & 30122.84 & 25584.42 & 30635.79 \\
\hline Cost of ADN2 & 7414.32 & 7434.06 & 7569.54 & 8968.73 \\
\hline Cost of ADN3 & 9281.99 & 9304.35 & 11038.58 & 8968.73 \\
\hline Cost of ADN4 & 9461.69 & 9486.69 & 9892.74 & 8968.73 \\
\hline Total cost of ADNs & 51460.95 & 56347.94 & 54085.28 & 57541.99 \\
\hline
\end{tabular}

Table 7 Cost of ADN with and without ESs

\begin{tabular}{llc}
\hline Cost & With ES (\$) & Without ES (\$) \\
\hline Cost of ADN1 & 24709 & 25302.95 \\
Cost of ADN2 & 7182.25 & 7414.32 \\
Cost of ADN3 & 8989.27 & 9281.99 \\
Cost of ADN4 & 9173.23 & 9461.69 \\
Total cost of ADNs & 50053.7 & 51460.95 \\
\hline
\end{tabular}

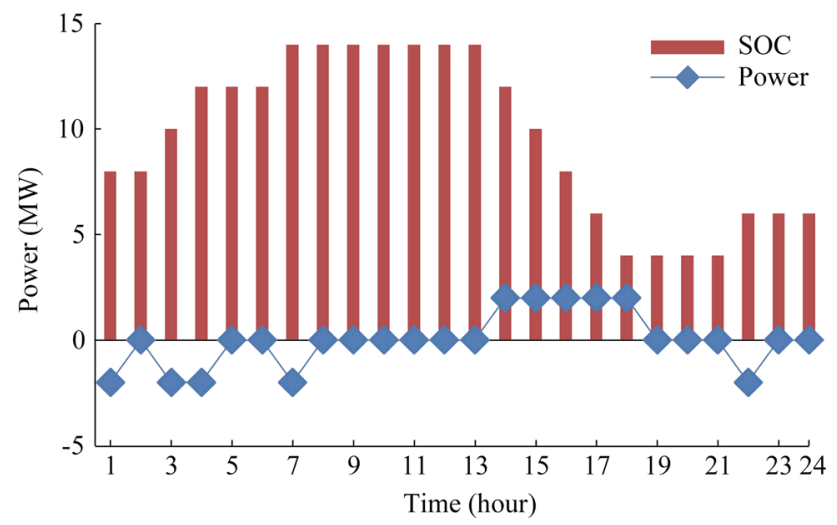

Fig. 9 Charging and discharging state of ES in ADN2

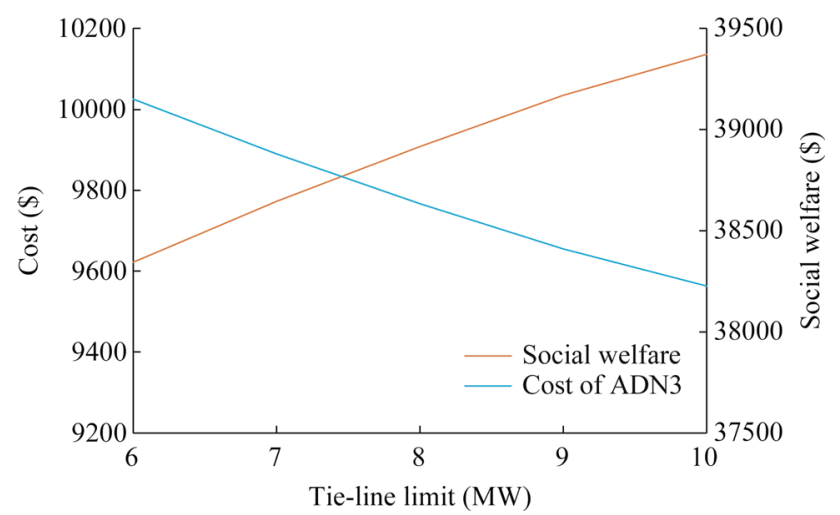

Fig. 10 Relationship between cost, social welfare and power limit based on multi-agent system. The process of market clearing in a bilateral electricity market incorporates the participation of ADNs while the dispatch of ADN is the generation schedule of DGs considering the impact of MCP. The main grid and ADNs affect each other through bid volume and MCP. The optimal solution of the whole system is obtained by multiple interactions between them. In this paper, we select a system with five suppliers, two buyers and four ADNs to verify the rationality of the algorithm compared with the schedule without interaction between ADNs and the main grid. Based on the analysis of ES and tie-line power limit, it can be known that the transmission power limit of tie-line is one of the important factors that affect the operating cost of ADNs. The usage of ES can reduce the cost of ADNs. Multi-energy complementary and coordinated dispatch can increase the overall interests of ADNs.

Acknowledgements This work was supported by The National High Technology Research and Development Program of China (No. 2014AA051902) and State Grid Science \& Technology Project (No. 5217L0140009).

Open Access This article is distributed under the terms of the Creative Commons Attribution 4.0 International License (http:// creativecommons.org/licenses/by/4.0/), which permits unrestricted use, distribution, and reproduction in any medium, provided you give appropriate credit to the original author(s) and the source, provide a link to the Creative Commons license, and indicate if changes were made.

\section{References}

[1] Hidalgo R, Abbey C, Joos G (2010) A review of active distribution networks enabling technologies. In: Proceedings of IEEE power and energy society general meeting, Providence, 25-29 July 2010, 9 pp

[2] McDonald J (2008) Adaptive intelligent power systems: active distribution networks. Energy Policy 36(12):4346-4351

[3] You S, Bindner HW, Hu JJ et al (2014) An overview of trends in distribution network planning: a movement towards smart 
planning. In: Proceedings of IEEE PES T\&D conference and exposition, Chicago, USA, 14-17 April 2014, 5 pp

[4] D'Adamo C, Jupe S, Abbey C (2009) Global survey on planning and operation of active distribution networks-update of CIGRE C6. 11 working group activities. In: Proceedings of electricity distribution-part 1, 2009. CIRED 2009. 20th international conference and exhibition on, Prague, Czech Republic, 8-11 June 2009, 4 pp

[5] You Y, Liu D, Zhong Q et al (2014) Research on optimal schedule strategy for active distribution network. Autom Electric Power Syst 38(9):177-183. doi:10.7500/AEPS20130804001

[6] Pilo F, Pisano G, Soma GG (2009) Advanced DMS to manage active distribution networks. In: Proceedings of IEEE PowerTech, Bucharest, Romania, 28 June-2 July 2009, 8 pp

[7] Borghetti A, Bosetti M, Grillo S et al (2010) Short-term scheduling and control of active distribution systems with high penetration of renewable resources. IEEE Syst J 4(3):313-322

[8] Zhao X, Chen Q, Xia Q et al (2013) Multi-period coordinated active-reactive scheduling of active distribution system. In: Proceedings of IEEE Power and Energy Society General Meeting (PES), Vancouver, Canada, 21-25 July 2013, 5pp

[9] Gill S, Kockar I, Ault GW (2014) Dynamic optimal power flow for active distribution networks. IEEE Trans Power Syst 29(1):121-131

[10] Yorino N, Hafiz HM, Sasaki Y et al (2012) High-speed real-time dynamic economic load dispatch. IEEE Trans Power Syst 27(2):621-630

[11] Gu Y, Xie L (2013) Early detection and optimal corrective measures of power system insecurity in enhanced look-ahead dispatch. IEEE Trans Power Syst 28(2):1297-1307

[12] Li Z, Wu W, Zhang B et al (2013) Dynamic economic dispatch using Lagrangian relaxation with multiplier updates based on a quasi-Newton method. IEEE Trans Power Syst 28(4):4516-4527

[13] Aguado JA, Quintana VH (2001) Inter-utilities power-exchange coordination: a market-oriented approach. IEEE Trans Power Syst 16(3):513-519

[14] Kim BH, Baldick R (1997) Coarse-grained distributed optimal power flow. IEEE Trans Power Syst 12(2):932-939

[15] Baldick R, Kim BH, Chase C et al (1999) A fast distributed implementation of optimal power flow. IEEE Trans Power Syst 14(3):858-864

[16] Kim BH, Baldick R (2000) A comparison of distributed optimal power flow algorithms. IEEE Trans Power Syst 15(2):599-604

[17] Bakirtzis AG, Biskas PN (2003) A decentralized solution to the DC-OPF of interconnected power systems. IEEE Trans Power Syst 18(3):1007-1013

[18] Biskas PN, Bakirtzis AG, Macheras NI et al (2005) A decentralized implementation of DC optimal power flow on a network of computers. IEEE Trans Power Syst 20(1):25-33

[19] Li Z, Wu W, Zhang B et al (2016) Decentralized multi-area dynamic economic dispatch using modified generalized benders decomposition. IEEE Trans Power Syst 31(1):526-538

[20] Ghasemi M, Aghaei J, Akbari E et al (2016) A differential evolution particle swarm optimizer for various types of multiarea economic dispatch problems. Energy 107(1):182-195

[21] Basu M (2013) Artificial bee colony optimization for multi-area economic dispatch. Int J Electr Power Energy Syst 49:181-187

[22] Basu M (2014) Teaching-learning-based optimization algorithm for multi-area economic dispatch. Energy 68(8):21-28

[23] Nogales FJ, Prieto FJ, Conejo AJ (1999) Multi-area AC optimal power flow: a new decomposition approach. In: Proceedings of the 13th power systems control conference (PSCC), Trondheim, Norway, June 1999, 6 pp

[24] Conejo AJ, Nogales FJ, Prieto FJ (2002) A decomposition procedure based on approximate Newton directions. Math Program 93(3):495-515
[25] Bakirtzis AG, Biskas PN (2003) A decentralized solution to the DC-OPF of interconnected power systems. IEEE Trans Power Syst 18(3):1007-1013

[26] Biskas PN, Bakirtzis AG, Macheras NI et al (2005) A decentralized implementation of DC optimal power flow on a network of computers. IEEE Trans Power Syst 20(1):25-33

[27] Nogales FJ, Prieto FJ, Conejo AJ (2003) A decomposition methodology applied to the multi-area optimal power flow problem. Ann Oper Res 120(1-4):99-116

[28] Hug-Glanzmann G, Andersson G (2009) Decentralized optimal power flow control for overlapping areas in power systems. IEEE Trans Power Syst 24(1):327-336

[29] Kargarian A, Fu Y (2014) System of systems based securityconstrained unit commitment incorporating active distribution grids. IEEE Trans Power Syst 29(5):2489-2498

[30] Li Z, Guo Q, Sun H et al (2016) Coordinated economic dispatch of coupled transmission and distribution systems using heterogeneous decomposition. IEEE Trans Power Syst 31(6):4817-4830

[31] Li Z, Guo Q, Sun H et al (2016) A new LMP-sensitivity-based heterogeneous decomposition for transmission and distribution coordinated economic dispatch. IEEE Trans Smart Grid. doi:10. 1109/TSG.2016.2571848

[32] Bessa RJ, Matos MA, Soares FJ et al (2012) Optimized bidding of a EV aggregation agent in the electricity market. IEEE Trans Smart Grid 3(1):443-452

[33] Zhou Z, Zhao F, Wang J (2011) Agent-based electricity market simulation with demand response from commercial buildings. IEEE Trans Smart Grid 2(4):580-588

[34] Li G, Shi J (2012) Agent-based modeling for trading wind power with uncertainty in the day-ahead wholesale electricity markets of single-sided auctions. Appl Energy 99(2):13-22

[35] Shafie-khah M, Catalão JPS (2015) A stochastic multi-layer agent-based model to study electricity market participants behavior. IEEE Trans Power Syst 30(2):867-881

[36] Yujiao L (2013) Short-term optimization and its risk management of renewable generations under different operating environments. Shanghai Jiaotong University, Shanghai

[37] Wang CS, Bowen H, Li G et al (2013) A general modeling method for optimal dispatch of combined cooling, heating and power microgrid. Proc CSEE 33(31):26-33

Jingzhe HU received the B.S. and M.S. degrees in electrical engineering from Northeast Dianli University and Tianjin University respectively. She is currently pursuing the Ph.D. degree in electrical engineering in Shanghai Jiao Tong University, Shanghai, China. Her research interests include power system optimization and power market.

Hao CONG received the B.S. degree in electrical engineering from Shanghai Jiao Tong University. He is currently pursuing the Ph.D. degree in electrical engineering in Shanghai Jiao Tong University, Shanghai, China. His research interests include power system optimization and power market.

Chuanwen JIANG received the M.S. and Ph.D degrees from Huazhong University of Science and Technology, Wuhan, China, in 1996 and 2000, respectively, and completed his postdoctoral research in the School of Electronic Information and Electrical Engineering, Shanghai Jiao Tong University, Shanghai, China, in 2002. He is a Professor with the School of Electronic Information and Electrical Engineering, Shanghai Jiao Tong University. $\mathrm{He}$ is currently researching reservoir dispatch, load forecast in power systems, and electrical power market. 